\title{
On a conjecture of Gustafsson and Lin concerning Laplacian growth
}

\author{
Stephen J. Gardiner ${ }^{1}\left[0 \cdot\right.$ Tomas Sjödin $^{2}[0$ \\ Received: 28 December 2021 / Revised: 28 December 2021 / Accepted: 9 January 2022 / \\ Published online: 25 January 2022 \\ (c) The Author(s) 2022
}

\begin{abstract}
Gustafsson and Lin recently published a significant result concerning Laplacian growth problems that start from a simply connected planar domain. However, the validity of their result depends on the verification of a particular conjecture. This paper provides the missing proof.
\end{abstract}

Keywords Laplacian growth $\cdot$ Partial balayage $\cdot$ Potential $\cdot$ Starshaped

Mathematics Subject Classification 31A15

\section{Introduction}

A recent book of Gustafsson and Lin [4] explores the evolution of domains under a Laplacian growth process that starts from a simply connected planar domain with smooth boundary. A key result of theirs, Theorem 5.1, states that this process can be continued indefinitely as a family of simply connected domains on a suitable branched Riemann surface. However, their theorem relies on the validity of a lemma which they believe to be true but are unable to prove. (See also section 8 of [3].) The purpose of this note is to verify their conjecture and so complete the proof of their result.

Let $g$ be a holomorphic function on a connected neighbourhood $\omega$ of $\overline{\mathbb{D}}$, where $\mathbb{D}$ denotes the unit disc, and let $\lambda$ denote planar Lebesgue measure. (We assume that

Harold S. Shapiro, in memoriam.

$凶$ Tomas Sjödin

tomas.sjodin@liu.se

Stephen J. Gardiner stephen.gardiner@ucd.ie

1 School of Mathematics and Statistics, University College Dublin, Dublin 4, Ireland

2 Department of Mathematics, Linköping University, 58183 Linköping, Sweden

Birkhäuser 
$g \not \equiv 0$ and assign $g$ the value 1 , say, outside $\omega$ to make it globally defined.) For each $t>0$ we define $\Omega(t)=\left\{u_{t}>0\right\}$, where

$$
u_{t}=\inf \left\{w \in C\left(\mathbb{R}^{2} \backslash\{0\}\right): w \geq 0, \Delta w \leq\left.|g|^{2} \lambda\right|_{\mathbb{R}^{2} \backslash \mathbb{D}}-t \delta_{0}\right\}
$$

in the sense of distributions and $\delta_{0}$ is the unit measure at 0 . The conjecture of Gustafsson and Lin is that the domains $\Omega(t)$ are simply connected for all sufficiently small $t>0$. Their difficulty in verifying it arises when the function $g$ has one or more zeros on $\partial \mathbb{D}$. Indeed, they remark that the same issue was also left unresolved in earlier work of Sakai [7]. We prove their conjecture below.

Theorem 1 There exists $\delta>0$ such that the domains $\Omega(t)(0<t<\delta)$ are all starshaped about 0 , and so in particular are simply connected.

Our proof of Theorem 1 remains valid if we replace $|g|^{2}$ in (1) by any $C^{1}$ function $f>0$ on a neighbourhood of $\overline{\mathbb{D}}$. (Indeed, with minor modifications, it also yields the corresponding result in higher dimensions for such functions $f$.) However, the result may fail if $f$ is allowed to have even one zero, as we now illustrate.

Example 2 There is a $C^{\infty}$ function $f: \mathbb{R}^{2} \rightarrow[0, \infty)$ with precisely one zero such that, if $|g|^{2}$ is replaced by $f$ in (1), then there are arbitrarily small values of $t>0$ for which $\Omega(t)$ is multiply connected.

Thus the geometrical character of $\Omega(t)$ for small $t>0$ is highly sensitive to the nature of this function $f$.

We will establish Theorem 1 and Example 2 in Sects. 3 and 4, respectively, following a brief review of the technique of partial balayage, on which these arguments rely. A survey of related topics, including quadrature domains and free boundary problems, may be found in [6].

\section{Partial balayage}

If $\mu$ is a (positive) measure with compact support in $\mathbb{R}^{2}$, then we define the logarithmic potential

$$
U \mu(x)=-\frac{1}{2 \pi} \int \log |x-y| d \mu(y) \quad\left(x \in \mathbb{R}^{2}\right)
$$

and note that $-\Delta U \mu=\mu$ (in the sense of distributions). Let $f: \mathbb{R}^{2} \rightarrow[0, \infty)$ be a continuous function such that $f \geq 1$ outside some compact set. The following construction, known as partial balayage, was developed by Gustafsson and Sakai [5] and also expounded by the authors in [2].

We define, for $t>0$,

$$
V_{t, f}=\sup \left\{v \in C\left(\mathbb{R}^{2} \backslash\{0\}\right):-\Delta v \leq\left. f \lambda\right|_{\mathbb{R}^{2} \backslash \mathbb{D}}, v \leq t U \delta_{0}\right\}
$$

and $u_{t, f}=t U \delta_{0}-V_{t, f}$, whence $u_{t, f} \geq 0$. Then

$$
-\Delta V_{t, f}=\left.f \lambda\right|_{\Omega_{f}(t) \backslash \mathbb{D}}, \quad \text { where } \quad \Omega_{f}(t)=\left\{u_{t, f}>0\right\} \supset \overline{\mathbb{D}},
$$


and so $V_{t, f}=U\left(\left.f \lambda\right|_{\Omega_{f}(t) \backslash \mathbb{D}}\right)$. It follows easily, using the assumption that $f \geq 1$ outside a compact set, that $\Omega_{f}(t)$ is bounded. Also,

$$
\int_{\Omega_{f}(t) \backslash \mathbb{D}} f(y) d \lambda(y)=t,
$$

since $t U \delta_{0}=V_{t, f}$ outside $\Omega_{f}(t)$.

Here are some more basic properties that we will need.

Proposition 3 Let $t>0$ and $f, f_{n}: \mathbb{R}^{2} \rightarrow[0, \infty)(n \geq 1)$ be continuous functions that exceed 1 outside some compact set.

(a) If $f_{1} \leq f_{2}$, then $V_{t, f_{1}} \leq V_{t, f_{2}}, u_{t, f_{1}} \geq u_{t, f_{2}}$ and $\Omega_{f_{2}}(t) \subset \Omega_{f_{1}}(t)$.

(b) If $\left(f_{n}\right)$ decreases to $f$, then $V_{t, f_{n}} \rightarrow V_{t, f}, u_{t, f_{n}} \rightarrow u_{t, f}$ and

$$
\cup_{n=1}^{\infty} \Omega_{f_{n}}(t)=\Omega_{f}(t) .
$$

(c) If $\left(f_{n}\right)$ increases to $f$, then $V_{t, f_{n}} \rightarrow V_{t, f}, u_{t, f_{n}} \rightarrow u_{t, f}$,

$$
\Omega_{f}(t) \subset \cap_{n=1}^{\infty} \Omega_{f_{n}}(t) \text { and } \int_{\cap_{n=1}^{\infty} \Omega_{f_{n}}(t) \backslash \Omega_{f}(t)} f d \lambda=0 \text {. }
$$

Proof (a) This follows immediately from the definition of $V_{t, f}$.

(b) By part (a) the sequence $\left(u_{t, f_{n}}\right)$, which equals $\left(t U \delta_{0}-U\left(\left.f_{n} \lambda\right|_{\Omega_{f_{n}}(t) \backslash \mathbb{D}}\right)\right)$, increases to the limit

$$
v=t U \delta_{0}-U\left(\left.f \lambda\right|_{\left.\left(\cup_{n} \Omega_{f_{n}}(t)\right) \backslash \mathbb{D}\right),}\right.
$$

where

$$
0 \leq v \leq u_{t, f}=t U \delta_{0}-U\left(\left.f \lambda\right|_{\Omega_{f}(t) \backslash \mathbb{D}}\right) .
$$

Since $v=u_{t, f}$ outside $\Omega_{f}(t)$, this equality must hold everywhere. The other assertions follow immediately.

(c) The argument is similar to part (b), except that $\left(\Omega_{f_{n}}(t)\right)$ is now decreasing.

Let

$$
D_{r}(w)=\{z \in \mathbb{C}:|z-w|<r\} \quad(w \in \mathbb{C}, r>0)
$$

and $D_{r}=D_{r}(0)$, so that $\mathbb{D}=D_{1}$. We identify $\mathbb{C}$ with $\mathbb{R}^{2}$ in the usual way. The function $g$ in Sect. 1 is holomorphic on a neighbourhood $\omega$ of $\overline{\mathbb{D}}$. We choose $R>1$ such that $\bar{D}_{R} \subset \omega$ and $g$ has no zeros in $\bar{D}_{R} \backslash \overline{\mathbb{D}}$. In the next section we choose $f$ such that $f=|g|^{2}$ on $\bar{D}_{R}$ and $f=1$ outside $D_{R+1}$, and will drop the symbol $f$ from the subscripts in the notation $V_{t, f}, u_{t, f}, \Omega_{f}(t)$ where no confusion can arise. We claim that there exists $\varepsilon>0$ such that

$$
\Omega(t) \subset D_{R} \quad(0<t<\varepsilon) .
$$

To see this we note that, if $1<r_{1}<r_{2}<R$, then there exists $c \in(0,1]$ such that $f \geq c$ on the set $A=\left(D_{r_{2}} \backslash D_{r_{1}}\right) \cup\left(\mathbb{R}^{2} \backslash D_{R+1}\right)$. Hence $\Omega_{f}(t) \subset \Omega_{c \chi_{A}}(t)$. The latter set is of the form $D_{\rho(t)}$ for some $\rho(t)>1$, and $\rho(t) \rightarrow r_{1}$ as $t \rightarrow 0+$, in view of (3). Indeed, there exists $r(t)>1$ such that $r(t) \rightarrow 1$ as $t \rightarrow 0+$ and $\Omega_{f}(t) \subset D_{r(t)}$. 


\section{Proof of Theorem 1}

Let $g, f$ and $R$ be as described above.

Lemma 4 Let $x_{1}, x_{2}, \ldots, x_{k}$ denote the zeros (if any) of $g$ on $\partial \mathbb{D}$. Then, for each $i \in\{1,2, \ldots, k\}$, there exist $r_{i} \in(0, R-1)$ and a positive constant $C_{i}$ such that

$$
\nabla f(x) \cdot x \geq-C_{i} f(x) \quad\left(x \in D_{r_{i}}\left(x_{i}\right) \backslash \overline{\mathbb{D}}\right) .
$$

Proof Suppose that $g$ has a zero of order $m$ at $x_{i}$. Then $f(x)=\left|x-x_{i}\right|^{2 m} h(x)$ on $\omega$, where $h \geq 0$ is smooth and $h\left(x_{i}\right)>0$. It follows that

$$
\begin{aligned}
\nabla f(x) \cdot x & =2 m\left|x-x_{i}\right|^{2 m-2} h(x)\left(x-x_{i}\right) \cdot x+\left|x-x_{i}\right|^{2 m} \nabla h(x) \cdot x \\
& =h(x)\left|x-x_{i}\right|^{2 m}\left(2 m \frac{\left(x-x_{i}\right) \cdot x}{\left|x-x_{i}\right|^{2}}+\frac{\nabla h(x) \cdot x}{h(x)}\right) \\
& \geq f(x) \frac{\nabla h(x) \cdot x}{h(x)} \quad\left(x \in D_{R} \backslash \overline{\mathbb{D}}\right),
\end{aligned}
$$

since

$$
\left(x-x_{i}\right) \cdot x=|x|^{2}-x_{i} \cdot x>0 \quad\left(|x|>\left|x_{i}\right|=1\right) .
$$

The result follows on noting that $h>0$ on a neighbourhood of $x_{i}$.

Lemma 5 There exists $C_{0}>0$ such that

$$
\nabla f(x) \cdot x+\left(C_{0}+2\right) f(x) \geq 0 \quad\left(x \in D_{R} \backslash \overline{\mathbb{D}}\right) .
$$

Proof Let $x_{i}, r_{i}, C_{i}(i=1, \ldots, k)$ be as in Lemma 4 and define

$$
A=D_{R} \backslash\left(\overline{\mathbb{D}} \cup D_{r_{1}}\left(x_{1}\right) \cup \cdots \cup D_{r_{k}}\left(x_{k}\right)\right) .
$$

Clearly $\inf _{A} f>0$. The result follows on choosing $C_{0}$ large enough so that $C_{0}+2 \geq$ $C_{i}(i=1, \ldots, k)$ and

$$
\inf _{x \in A} \nabla f(x) \cdot x+\left(C_{0}+2\right) \inf _{A} f \geq 0 .
$$

Proof of Theorem 1 Let

$$
v_{t}(x)=\nabla u_{t}(x) \cdot x+C_{0} u_{t}(x) \quad(t>0)
$$

where $u_{t}$ is as in Sect. 2 and $C_{0}$ is as in Lemma 5. We choose $R>1$ and $\varepsilon>0$ as in Sect. 2, whence $\Omega(t) \subset D_{R}$ when $0<t<\varepsilon$. Since

$$
\begin{aligned}
\Delta\left(\nabla u_{t}(x) \cdot x\right) & =2 \Delta u_{t}(x)+\left(\nabla \Delta u_{t}(x)\right) \cdot x \\
& =2 f(x)+\nabla f(x) \cdot x \quad(x \in \Omega(t) \backslash \overline{\mathbb{D}}),
\end{aligned}
$$


the function $v_{t}$ is subharmonic in $\Omega(t) \backslash \overline{\mathbb{D}}$.

We know that $u_{t}$, and hence $v_{t}$, vanishes outside $\Omega(t)$. Next, we will show that $v_{t} \leq 0$ on $\partial \mathbb{D}$ for all sufficiently small $t$. Suppose that $x \neq 0$. Since

$$
u_{t}(x)=-\frac{t}{2 \pi} \log |x|+\frac{1}{2 \pi} \int_{\Omega(t) \backslash \mathbb{D}} \log |x-y| f(y) d \lambda(y),
$$

we see that

$$
\begin{aligned}
\nabla u_{t}(x) \cdot x= & -\frac{t}{2 \pi} \frac{x}{|x|^{2}} \cdot x+\frac{1}{2 \pi} \int_{\Omega(t) \backslash \mathbb{D}} \frac{x-y}{|x-y|^{2}} \cdot x f(y) d \lambda(y) \\
= & -\frac{t}{2 \pi}+\frac{1}{2 \pi} \int_{\Omega(t) \backslash \mathbb{D}} \frac{x-y}{|x-y|^{2}} \cdot(x-y) f(y) d \lambda(y) \\
& +\frac{1}{2 \pi} \int_{\Omega(t) \backslash \mathbb{D}} \frac{x-y}{|x-y|^{2}} \cdot y f(y) d \lambda(y) \\
= & \frac{1}{2 \pi} \int_{\Omega(t) \backslash \mathbb{D}} \frac{x-y}{|x-y|^{2}} \cdot y f(y) d \lambda(y),
\end{aligned}
$$

by (3). This last integrand is negative when $|x|=1$, since $(x-y) \cdot y=x \cdot y-|y|^{2}$ and $|y|>1$. Let

$$
A_{x, t}=\{y \in \Omega(t) \backslash \mathbb{D}: x \cdot y \leq 0\} \quad(x \in \partial \mathbb{D}, t>0) .
$$

Then

$$
\frac{x-y}{|x-y|^{2}} \cdot y \leq-\frac{|y|^{2}}{|x-y|^{2}} \leq-\frac{1}{4} \quad\left(y \in A_{x, t}\right)
$$

and so

$$
\int_{\Omega(t) \backslash \mathbb{D}} \frac{x-y}{|x-y|^{2}} \cdot y f(y) d \lambda(y) \leq-\frac{1}{4} \int_{A_{x, t}} f d \lambda \leq-\frac{1}{4} \inf _{z \in \partial \mathbb{D}} \int_{A_{z, t}} f d \lambda .
$$

There exists $c>0$ such that $\Omega(t) \supset D_{1+c t}$, because $f$ is bounded above. Since $f$ has only finitely many zeros on $\partial \mathbb{D}$, there exists $C_{*}>0$ such that

$$
\inf _{z \in \partial \mathbb{D}} \int_{A_{z, t}} f d \lambda \geq C_{*} t \quad(0<t<\varepsilon)
$$

so we now see from (5) and (6) that

$$
\nabla u_{t}(x) \cdot x \leq-\frac{C_{*}}{8 \pi} t<0 \quad(x \in \partial \mathbb{D}, 0<t<\varepsilon)
$$


Also, it follows from (4) and (3) that the family $\left\{u_{t} / t: 0<t<\varepsilon\right\}$ of subharmonic functions on $\mathbb{R}^{2} \backslash\{0\}$ is locally uniformly bounded above. Since

$$
\limsup _{t \rightarrow 0+} \frac{u_{t}(x)}{t}=0 \quad\left(x \in \mathbb{R}^{2} \backslash \overline{\mathbb{D}}\right),
$$

this upper limit is bounded above by $-(\log |x|) / 2 \pi$ on $\overline{\mathbb{D}}$. It follows from Corollary 5.7 .2 of [1] that $u_{t}(x) / t \rightarrow 0$ uniformly on $\partial \mathbb{D}$ as $t \rightarrow 0+$. Hence, by (7), there exists $\delta \in(0, \varepsilon)$ such that

$$
\nabla u_{t}(x) \cdot x \leq-\frac{C_{*}}{8 \pi} \frac{t}{u_{t}(x)} u_{t}(x) \leq-C_{0} u_{t}(x) \quad(x \in \partial \mathbb{D}, 0<t<\delta),
$$

and so $v_{t} \leq 0$ on $\partial \mathbb{D}$ when $0<t<\delta$, as claimed.

We can now apply the maximum principle to the subharmonic function $v_{t}$ on $\Omega(t) \backslash \overline{\mathbb{D}}$ to see that $v_{t}<0$ there. Hence

$$
\nabla u_{t}(x) \cdot x \leq-C_{0} u_{t}(x)<0 \quad(x \in \Omega(t) \backslash \overline{\mathbb{D}}, 0<t<\delta),
$$

and we also know that $\nabla u_{t}(x) \cdot x=0$ on $\mathbb{R}^{2} \backslash \Omega(t)$. Since $\overline{\mathbb{D}} \subset\left\{u_{t}>0\right\}=\Omega(t)$, and $u_{t}$ is decreasing in the radial direction from 0 at each point of $\Omega(t) \backslash \mathbb{D}$, it follows that $\Omega(t)$ is starshaped about 0 , as required.

\section{Details of Example 2}

Let

$$
f_{e}(x)=\left\{\begin{array}{cc}
\exp \left(-\left|x-y_{0}\right|^{-2}\right) & \left(x \in \mathbb{R}^{2} \backslash\left\{y_{0}\right\}\right) \\
0 & \left(x=y_{0}\right)
\end{array},\right.
$$

where $y_{0}$ is the point $(1,0)$, and let $\psi: \mathbb{R}^{2} \rightarrow[0,1]$ be a $C^{\infty}$ function such that $\psi(x)=0$ when $|x| \in\left[\frac{1}{2}, \frac{3}{4}\right]$ and $\psi(x)=1$ when $|x| \in\left[0, \frac{1}{4}\right] \cup[1, \infty)$. For each $n$ in $\mathbb{N}$ we define

$$
x_{n}=\left(\cos \frac{\pi}{n}, \sin \frac{\pi}{n}\right) \text { and } r_{n}=\frac{1}{n(n+1)},
$$

whence the discs $\bar{D}_{r_{n}}\left(x_{n}\right)$ are pairwise disjoint, and the closed annulus

$$
A_{n}=\bar{D}_{3 r_{n} / 4}\left(x_{n}\right) \backslash D_{r_{n} / 2}\left(x_{n}\right) \text {. }
$$

We further define

$$
\psi_{n}(x)=\psi\left(\frac{x-x_{n}}{r_{n}}\right), \quad \psi_{n, m}(x)=\frac{\psi_{n}(x)+1 / m}{1+1 / m} \quad(m \in \mathbb{N})
$$

and

$$
f_{0}=f_{e} \prod_{n \geq 1} \psi_{n}
$$


Since $\int_{\Omega_{f_{0}}(t) \backslash D_{1}} f_{0} d \lambda=t$ and

$$
\int_{D_{r_{1} / 4}\left(x_{1}\right) \backslash D_{1}} f_{0} d \lambda=\int_{D_{r_{1} / 4}\left(x_{1}\right) \backslash D_{1}} f_{e} d \lambda>0,
$$

we can choose $t_{1}>0$ small enough to ensure that

$$
D_{r_{1} / 4}\left(x_{1}\right) \backslash \Omega_{f_{0}}\left(t_{1}\right) \neq \emptyset
$$

In view of (2) the nonnegative function $u_{t_{1}}, f_{0}$ is nonconstant and harmonic on the domain $\left(D_{1} \cup A_{1}^{\circ}\right) \backslash\{0\}$, and so is strictly positive there. Further, $u_{t_{1}, f_{0}}$ cannot take the value 0 at any point $y$ of $\partial A_{1}$, since this would imply that $\nabla u_{t_{1}}, f_{0}(y)=0$, which contradicts the Hopf lemma. Hence

$$
A_{1} \subset \Omega_{f_{0}}\left(t_{1}\right)
$$

and the constant $c_{1}=\left(\inf _{A_{1}} u_{t_{1}, f_{0}}\right) / 2$ is strictly positive. We define

$$
f_{1, m}=f_{e} \psi_{1, m} \prod_{n \geq 2} \psi_{n} \quad(m \in \mathbb{N})
$$

and note that the sequence $\left(f_{1, m}\right)$ decreases to $f_{0}$, whence by Proposition 3 the sequences $\left(\Omega_{f_{1}, m}\left(t_{1}\right)\right)$ and $\left(u_{t_{1}, f_{1, m}}\right)$ are increasing,

$$
\lim _{m \rightarrow \infty} u_{t_{1}, f_{1, m}}=u_{t_{1}, f_{0}} \quad \text { and } \cup_{m} \Omega_{f_{1}, m}\left(t_{1}\right)=\Omega_{f_{0}}\left(t_{1}\right)
$$

By compactness we can choose $m_{1} \in \mathbb{N}$ such that $A_{1} \subset \Omega_{f_{1}, m_{1}}\left(t_{1}\right)$ and $\inf _{A_{1}} u_{t, f_{1, m_{1}}}>c_{1}$, and then define

$$
f_{1}=f_{1, m_{1}}=f_{e} \psi_{1, m_{1}} \prod_{n \geq 2} \psi_{n}
$$

Since $f_{1} \geq f_{0}$ we note that

$$
D_{r_{1} / 4}\left(x_{1}\right) \backslash \Omega_{f_{1}}\left(t_{1}\right) \supset D_{r_{1} / 4}\left(x_{1}\right) \backslash \Omega_{f_{0}}\left(t_{1}\right) \neq \emptyset .
$$

Next, arguing as above, we choose $t_{2} \in\left(0, t_{1} / 2\right)$ small enough to ensure that

$$
D_{r_{2} / 4}\left(x_{2}\right) \backslash \Omega_{f_{1}}\left(t_{2}\right) \neq \varnothing
$$

and, noting that $f_{1}=f_{0}$ outside $D_{r_{1}}\left(x_{1}\right)$, observe that

$$
A_{2} \subset \Omega_{f_{1}}\left(t_{2}\right)
$$


Let $c_{2}$ denote the positive constant $\left(\inf _{A_{2}} u_{t_{2}}, f_{1}\right) / 2$. We define

$$
f_{2, m}=f_{e} \psi_{1, m_{1}} \psi_{2, m} \prod_{n \geq 3} \psi_{n} \quad(m \in \mathbb{N})
$$

and note that $\left(f_{2, m}\right)$ decreases to $f_{1}$. As before, we can choose $m_{2} \in \mathbb{N}$ such that

$$
A_{j} \subset \Omega_{f_{2}, m_{2}}\left(t_{j}\right) \text { and } \inf _{A_{j}} u_{t_{j}, f_{2, m_{2}}}>c_{j} \quad(j=1,2) .
$$

We define

$$
f_{2}=f_{2, m_{2}}=f_{e} \psi_{1, m_{1}} \psi_{2, m_{2}} \prod_{n \geq 3} \psi_{n}
$$

and note that $\Omega_{f_{2}}(t) \subset \Omega_{f_{1}}(t)(t>0)$, whence

$$
D_{r_{1} / 4}\left(x_{1}\right) \backslash \Omega_{f_{2}}\left(t_{1}\right) \neq \emptyset \text { and } \quad D_{r_{2} / 4}\left(x_{2}\right) \backslash \Omega_{f_{2}}\left(t_{2}\right) \neq \emptyset \text {. }
$$

Proceeding inductively in this way, we obtain a sequence of numbers $\left(t_{j}\right)$ decreasing to 0 , a sequence of positive numbers $\left(c_{j}\right)$, and an increasing sequence of functions $\left(f_{k}\right)$ such that

$$
A_{j} \subset \Omega_{f_{k}}\left(t_{j}\right), \quad D_{r_{j} / 4}\left(x_{j}\right) \backslash \Omega_{f_{k}}\left(t_{j}\right) \neq \emptyset \quad \text { and } \quad u_{t_{j}, f_{k}}>c_{j} \quad \text { on } A_{j} \quad(1 \leq j \leq k) \text {. }
$$

We define

$$
f=\lim _{j \rightarrow \infty} f_{j}=f_{e} \prod_{j \geq 1} \psi_{j, m_{j}}
$$

Clearly

$$
D_{r_{j} / 4}\left(x_{j}\right) \backslash \Omega_{f}\left(t_{j}\right) \neq \emptyset \quad(j \in \mathbb{N}) .
$$

By Proposition 3 again we note that $\left(u_{t, f_{k}}\right)$ decreases to $u_{t, f}$ as $k \rightarrow \infty$ for every $t>0$. Since $u_{t_{j}, f_{k}} \geq c_{j}$ on $A_{j}$ for all $j \leq k$, we see that $u_{t_{j}, f} \geq c_{j}$ on $A_{j}$ for all $j$, and so $A_{j} \subset \Omega_{f}\left(t_{j}\right)$ for each $j$. Thus $\Omega_{f}\left(t_{j}\right)$ is multiply connected for each $j \in \mathbb{N}$. Finally, $f$ vanishes precisely at $y_{0}$ and, since

$$
\inf \left\{\frac{r_{j}}{\left|x-y_{0}\right|^{2}}: x \in D_{r_{j}}\left(x_{j}\right), j \geq 1\right\}>0,
$$

we see that $f \in C^{\infty}\left(\mathbb{R}^{2}\right)$.

Funding Open access funding provided by Linköping University.

Data availability Data sharing is not applicable to this article as no datasets were generated or analysed during the current study.

\section{Declarations}

Conflict of interest The authors did not receive support from any organization for the submitted work. The authors have no competing interests to declare that are relevant to the content of this article. 
Open Access This article is licensed under a Creative Commons Attribution 4.0 International License, which permits use, sharing, adaptation, distribution and reproduction in any medium or format, as long as you give appropriate credit to the original author(s) and the source, provide a link to the Creative Commons licence, and indicate if changes were made. The images or other third party material in this article are included in the article's Creative Commons licence, unless indicated otherwise in a credit line to the material. If material is not included in the article's Creative Commons licence and your intended use is not permitted by statutory regulation or exceeds the permitted use, you will need to obtain permission directly from the copyright holder. To view a copy of this licence, visit http://creativecommons.org/licenses/by/4.0/.

\section{References}

1. Armitage, D.H., Gardiner, S.J.: Classical Potential Theory. Springer, London (2001)

2. Gardiner, S.J., Sjödin, T.: Partial balayage and the exterior inverse problem of potential theory. In: Potential Theory and Stochastics in Albac, Bucharest, Theta, pp. 111-123 (2009)

3. Gustafsson, B.: Laplacian growth on a branched Riemann surface. In: Analysis on Shapes of Solutions to Partial Differential Equations, RIMS, Kokyuroku No. 2082, pp. 145-161 (2018)

4. Gustafsson, B., Lin, Y.-L.: Laplacian growth on branched Riemann surfaces. Lecture Notes in Mathematics, vol. 2287. Springer, Cham (2021)

5. Gustafsson, B., Sakai, M.: Properties of some balayage operators, with applications to quadrature domains and moving boundary problems. Nonlinear Anal. 22, 1221-1245 (1994)

6. Gustafsson, B., Shapiro, H.S.: What is a quadrature domain? In: Quadrature Domains and their Applications, vol. 156. Oper. Theory Adv. Appl. Birkhäuser, Basel, pp. 1-25 (2005)

7. Sakai, M.: Finiteness of the family of simply connected quadrature domains. In: Potential Theory (Prague, 1987). Plenum, New York, pp. 295-305 (1988)

Publisher's Note Springer Nature remains neutral with regard to jurisdictional claims in published maps and institutional affiliations. 